\title{
Single exponential decay waveform; a synergistic combination of electroporation and electrolysis (E2) for tissue ablation
}

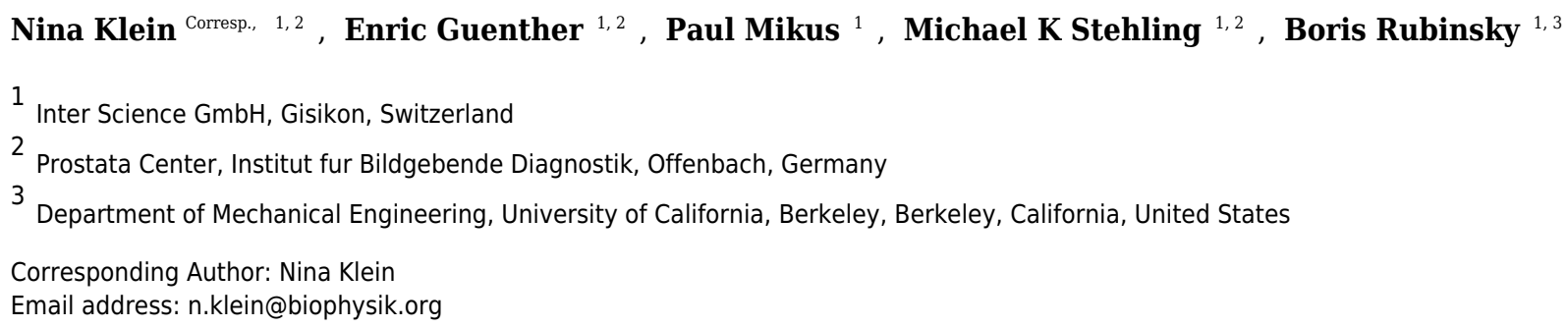

Background: Electrolytic ablation and electroporation based ablation are minimally invasive, nonthermal surgical technologies that employ electrical currents and electric fields to ablate undesirable cells in a volume of tissue. In this study we explore the attributes of a new tissue ablation technology that simultaneously delivers a synergistic combination of electroporation and electrolysis (E2).

Method: A new device that delivers a controlled dose of electroporation field and electrolysis currents in the form of a single exponential decay waveform (EDW), was applied to the pig liver and the effect of various parameters on the extent of tissue ablation was examined with histology.

Results: Histological analysis shows that E2 delivered as EDW can produce tissue ablation in volumes of clinical significance, using electrical and temporal parameters which, if used in electroporation or electrolysis separately, cannot ablate the tissue

Discussion: The E2 combination has advantages over the three basic technologies of non-thermal ablation: electrolytic ablation, electrochemical ablation (reversible electroporation with injection of drugs) and irreversible electroporation. E2 ablates clinically relevant volumes of tissue in a shorter period of time than electrolysis and electroporation, without the need to inject drugs as in reversible electroporation or use paralyzing anesthesia as in irreversible electroporation. 
1 Single exponential decay waveform; a synergistic combination of electroporation and electrolysis (E2) for tissue ablation

3 Nina Klein ${ }^{1,2^{*}}$, Enric Gunther ${ }^{1,2^{*}}$, Paul Mikus ${ }^{1}$, Michael Stehling ${ }^{1,2}$, Boris Rubinsky ${ }^{1,3}$

$4 \quad{ }^{1}$ Inter Science GmbH, Biophysics, 6038 Gisikon, Luzern, Switzerland

$5 \quad{ }^{2}$ Institut fur bildgebende Diagnostik, Prostata Center, 63067 Offenbach, Germany

$6{ }^{3}$ Department of Mechanical Engineering, University of California Berkeley, Berkeley CA 84720

7 USA

8 Corresponding Author: Nina Klein

9 E-mail address - n.klein@biophysik.org 
10

11

12

13

14

15

16

17

18

19

20

21

22

23

24

25

26

27

28

\section{Abstract}

Background: Electrolytic ablation and electroporation based ablation are minimally invasive, non-thermal surgical technologies that employ electrical currents and electric fields to ablate undesirable cells in a volume of tissue. In this study we explore the attributes of a new tissue ablation technology that simultaneously delivers a synergistic combination of electroporation and electrolysis (E2).

Method: A new device that delivers a controlled dose of electroporation field and electrolysis currents in the form of a single exponential decay waveform (EDW), was applied to the pig liver and the effect of various parameters on the extent of tissue ablation was examined with histology.

Results: Histological analysis shows that E2 delivered as EDW can produce tissue ablation in volumes of clinical significance, using electrical and temporal parameters which, if used in electroporation or electrolysis separately, cannot ablate the tissue

Discussion: The E2 combination has advantages over the three basic technologies of nonthermal ablation: electrolytic ablation, electrochemical ablation (reversible electroporation with injection of drugs) and irreversible electroporation. E2 ablates clinically relevant volumes of tissue in a shorter period of time than electrolysis and electroporation, without the need to inject drugs as in reversible electroporation or use paralyzing anesthesia as in irreversible electroporation.

\section{Introduction}

A number of biophysical and biochemical phenomena occur simultaneously when electric fields are applied across biological matter. These include Joule heating due to electrical current energy dissipation, electrolytic reactions at the interface between the electrodes and the biological milieu, and cell membrane permeabilization known as electroporation. All these electrical phenomena are used for tissue ablation. Usually the electrical potential delivery protocol is designed in such a way as to maximize one phenomenon, while minimizing the others. For example, in non-thermal irreversible electroporation (NTIRE) the electrical potential profile is designed to maximize irreversible electroporation while minimizing Joule heating (Davalos et al. 2005). The non-thermal aspect of NTIRE was found to be beneficial in tissue ablation treatments, in which it is desired to spare vital sites in the treated lesion, such as blood vessels and nerves.

In electrolytic tissue ablation, cell death is caused by the chemical interaction between the products of electrolysis and cells (Nilsson et al. 2000),(Czymek et al. 2011). Because the ablation is caused by a chemical reaction, it is a function of compounds concentration and time of exposure. One drawback of tissue ablation by electrolysis is the need for high concentrations of electrolytes and lengthy times of exposure. An advantage is the very low currents and voltages used.

In ablation by electroporation, brief, pulsed, high electric fields are used to permeabilize the cell membrane. Lower electric fields and small numbers of pulses yield reversible electroporation, in which the cell membrane permeabilization is temporary. Higher electric fields with larger number of pulses yield irreversible electroporation in which the cell membrane permeabilization 
51 is permanent, which results in cell death. Both reversible and irreversible electroporation are 52 used for tissue ablation, each with their advantages and disadvantages. Reversible tissue 53 electroporation is used for tissue ablation in combination with cytotoxic additives, in a 54 procedure known as electrochemotherapy (Mir, Belehradek et al. 1991), (Marty, Sersa et al. 55 2006). One advantage of ablation by means of irreversible electroporation over 56 electrochemotherapy is that no chemotoxic drugs are injected into the tissue (Rubinsky et al. 2007), while the advantage of electrochemotherapy over irreversible electroporation is the use of fewer pulses and lower electric fields. The need to inject cytotoxic additives adds a complicating step to the electrochemotherapy procedure. Cell death through electrochemotherapy is dependent on mitosis cycle rendering and is possibly more tissue selective (Mir, Banoun, Paoletti 1988), (Orlowski et al. 1988), while irreversible electroporation induces apoptosis and necrosis instantaneously over the whole volume exposed to sufficiently high fields. However, the high electric fields and the large number of pulses used in conventional irreversible electroporation protocols cause some undesirable effects. They induce muscle contractions that require the use of a muscle relaxant and deep anesthesia during surgery. Every clinical electroporation protocols, reversible or irreversible, generates some products of electrolysis, and some heat (Turjanski et al. 2011)(Maglietti et al. 2013). We have recently shown that if substantial amounts of products of electrolysis are inadvertently generated during an electroporation protocol, a highly detrimental electrical discharge across the layer of gas formed on the electrodes can occur (Guenther et al. 2015).

In several recent papers we have shown that combining electroporation and electrolysis (E2) sagaciously yields a new technology of tissue ablation with certain advantages over tissue ablation by electroporation (reversible or irreversible) or electrolysis alone (Phillips, Raju et al. 2015) (Phillips et al. 2016) (Stehling et al. 2016). We have developed several possible synergistic electroporation and electrolysis (E2) protocols. One effective combination entails delivering first several (eight) reversible electroporation type pulses followed by the injection of a low voltage direct current to generate products of electrolysis. While effective, this combination requires two different power supplies, one for electroporation and the second for electrolysis (Phillips et al. 2015) (Phillips et al. 2016) (Stehling et al. 2016). The combined voltage profile of electroporation pulses followed by low voltage electrolysis reminded us of an exponential decay waveform (EDW), generated by the discharge of a capacitor; a type of pulse which was rather common in the early stages of electroporation research (Sale and Hamilton 1967). The shape of the capacitor discharge exponential decay waveform is a high initial voltage followed by a rapid decay towards a trailing low voltage. This type of waveform is still used in cell electroporation. We thought that with a properly chosen set of capacitor discharge parameters, the initial high voltage over a suitable timeframe could serve for electroporation, while the trailing lower voltage could generate sufficient charge for the generation of electrolytic products. The feasibility of tissue ablation with a EDW, was shown in the liver of a small rodent (Phillips et al. 2016).

The goal of this study was to extend the observations made in a small animal model and in an acute study (Phillips, Krishnan et al. 2016) to a larger animal model and a chronic study in order to show that EDW has the ability to ablate tissue volumes of clinical significance. The experimental study was supported by a first order mathematical analysis to evaluate the electric fields and extent of thermal damage generated by the exponential decay waveform. 


\section{Materials and methods:}

Animal protocol:

The study was approved by Sir José Antonio Rodríguez Correa, Director of Animal Health Programs and General Director of Department of Agriculture and Livestock, Ministry of Environment and Rural, Agricultural Policies and Territory, Government of Autonomous Community of Extremadura (Spain), with application form number: 2015209030009567 and study register number: 100370001499 . The experiment was conducted on in vivo pig liver, which was in accordance with Royal Decree Law 53/2013 (Feb.1st). According to the study protocol, three female pigs between $90-110 \mathrm{~kg}$ were treated. After being fasted for 24 hours, animals were pre-medicated with a combination of diazepam $(0.4 \mathrm{mg} / \mathrm{kg})$ and ketamine $(15 \mathrm{mg} / \mathrm{kg})$ injected intramuscularly (IM). Anesthesia was induced with intravenous (IV) Propofol (3mg/kg). Endotracheal intubation was performed and anesthesia was maintained with sevoflurane in oxygen (adjusted to 1.8-2\% End tidal sevoflurane). Possible postoperative pain was treated with Buprenorphine $0.01 \mathrm{mg} / \mathrm{kg} \mathrm{IM}$ Pre-med at recovery and Carprofen $4 \mathrm{mg} / \mathrm{kg}$ at extubation/recovery. Cefazolin $25 \mathrm{mg} / \mathrm{kg}$ IV was administrated every 2 hours. If found to be needed during the procedure, the study had the ability to deliver pancuronium $(0.1 \mathrm{mg} / \mathrm{kg}$, at a dose of $1 \mathrm{mg} / \mathrm{ml}$ ) through an IV to reduce muscle contractions during the application of the electrical pulses. The liver was exposed via a midline incision. The treatment was delivered using two 18-gauge Titanium needles (Inter Science $\mathrm{GmbH}$, Ch) with a variable length $(1-4 \mathrm{~cm}$ exposed treatment length) insulating sheath inserted in the liver. Titanium was chosen, because, unlike steel or aluminum it is chemically inert, is biocompatible and at room temperature inert to oxygen, chloride and corrosion (Emsley 2001). The 18-gauge $(1.02 \mathrm{~mm})$ variable length electrodes were custom designed for the delivery of both electroporation and electrolytic pulse sequences.

The experiment was carried out in an open-surgery setting to maximize the availability of liver compartments. The delivery of the single exponential decay waveform solely took place through the described needle-type electrodes. This type of intervention in which only probes are inserted in tissue for ablation is defined as minimally invasive, as opposed to invasive resection surgery. This definition applies to cryosurgery, radiofrequency ablation and the various electroporation modalities.

Two electrodes were inserted in the liver under ultrasound monitoring, in a roughly axial parallel configuration, normal to the liver surface. Ultrasound images were also taken throughout the procedure. Since no apparatus is currently available to produce the exponential decay voltage waveform needed for the SEE procedure conceived by us, we have designed and built a new power supply described in the following device section. The parameters varied in this study were: the initial voltage and the time constants of the exponential voltage waveform. In addition, we varied the number of exponential voltage waveforms delivered. A total of 23 lesions were produced, in three pigs, in separate experiments. Animals were sacrificed at 24 hours. The pigs were euthanized using Euthasol $2.2 \mathrm{ml} / \mathrm{kg} \mathrm{IV}$. 
134 To fix the liver for microscopic viewing, a Foley catheter was placed into the descending aorta 135 and the hepatic vein was snipped off for drainage of the affluent. The liver was flushed with 136 physiological saline for ten minutes at a hydrostatic pressure of $80 \mathrm{mmHg}$ from a pressurized IV 137 drip. Immediately following saline perfusion, a $10 \%$ formalin fixative was perfused in the same 138 way for ten minutes. The liver lobe in which the SEE lesion was made was removed and stored in 139 the same formalin solution. For microscopic analysis, the tissue was bread loafed perpendicular 140 to the capsule surface and parallel to the needle tracts. The needle tracts were marked on the 141 liver surface and the exact location of each experiment was noted to be able to find the correct 142 needle tracts. All cassettes were processed routinely from 10\% phosphate buffered formalin to 143 wax blocks. Five micrometer sections were made from each block and stained with Masson's 144 trichromatic stain for histologic examination. The stained samples were examined and analyzed 145 by an independent histology service company and reports were prepared (Narayan Raju, Inc, 146 South San Francisco, CA). The focus of the histology was to verify the extent and nature of tissue 147 ablation with E2. To produce information of practical clinical value, the focus of the analysis was 148 on verifying the ability to produce a continuous lesion between the electrodes.

\section{Device}

150

151

152

153

154

155

156

157

158

159

160

161

162

163

164

We were unable to find a power supply that can produce the waveform parameters, required for an EDW protocol in tissues with the dimensions of the pig liver. Therefore, we designed a power supply that operates in the modality of capacitor discharge electroporation systems (e.g. Gene Pulser Xcell ${ }^{\mathrm{TM}}$ Electroporation System, BioRad, Hercules, CA) with an enhanced performance. The conventional type capacitors used were replaced with a 100 microfarad capacitor to provide the charge required for electrolysis. Similar to the Gene Pulser Xcell ${ }^{\mathrm{TM}}$, the generator has an output of up to $3 \mathrm{kV}$. Because of the larger capacitors it can generate exponential decay waveforms up to time ranges of hundred milliseconds, depending on tissue conductivity and thereby simultaneously deliver electrolysis and electroporation. The apparatus selects and matches the internal components needed to produce the time constants selected for the specific tissue conductivity of the treatment area by selecting an appropriate capacitor. The apparatus is able to produce and deliver the exponential decay voltage profile in the time and voltage range for the specific treatment area. Figure 1B illustrates typical exponential decays shapes obtained for the in Table 1 listed electrical parameters of resistance and capacitance. Resistance and capacitance fully define the electrical components of the device.

Initial Voltage U0

Exposure length

Decay

Power supply capacitance

Distance between electrodes

Electrode diameter

Liver: electrical conductivity

Liver: heat capacity

Liver: density

Liver: thermal conductivity

Titanium: electrical conductivity
750V (Fig.3), 1000V (Fig.4,5), 1500V (Fig.6)

$1 \mathrm{~cm}$ (Fig.3,4,5) 2cm (Fig.6)

Exponential capacitor discharge

Listed in Figures

$1.5 \mathrm{~cm}$

$1 \mathrm{~mm}$

$0.286 \mathrm{~S} / \mathrm{m}$

$3750 \mathrm{~J} /\left(\mathrm{kg}^{*} \mathrm{~K}\right)$

$1000 \mathrm{~kg} / \mathrm{m}^{\wedge} 3$

$0.52 \mathrm{~W} /\left(\mathrm{m}^{*} \mathrm{~K}\right)$

$7.4 \mathrm{e} 5 \mathrm{~S} / \mathrm{m}$ 
Titanium: heat capacity

Titanium: density

Titanium: thermal conductivity $\mathrm{k}$
$710 \mathrm{~J} /\left(\mathrm{kg}^{*} \mathrm{~K}\right)$

$4940 \mathrm{~kg} / \mathrm{m} 3$

$7.5 \mathrm{~W} /\left(\mathrm{m}^{*} \mathrm{~K}\right)$

165 Table 1: Parameters used for the thermal and electrical field calculations. 


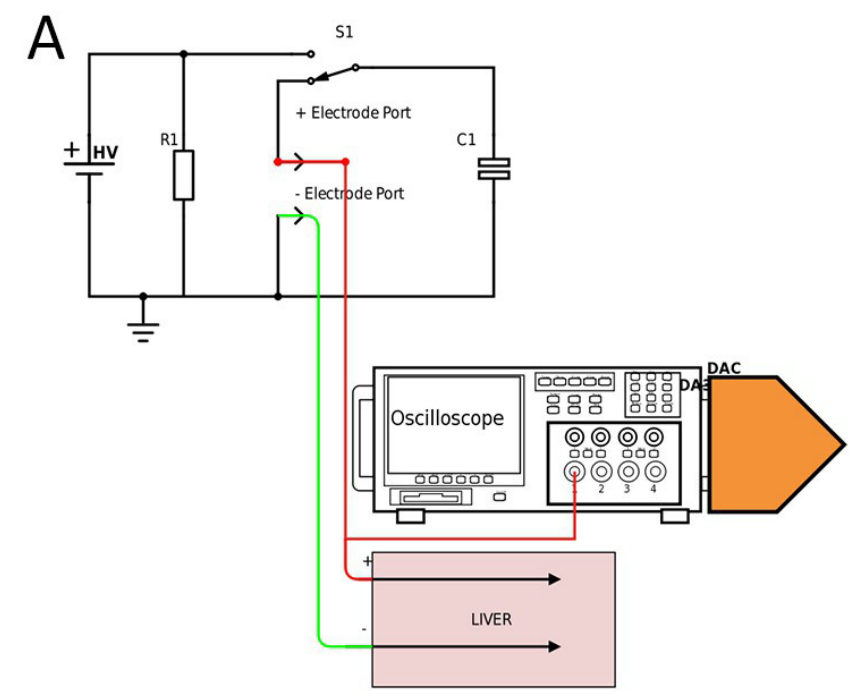

Peak Voltage: $1.0039 \mathrm{kV}$
Time till Voltage dropped below $1 / \mathrm{E}(\mathrm{Tau}): 37.1724 \mathrm{~ms}$
Time till Voltage dropped below $450 \mathrm{~V}(300 \mathrm{~V} / \mathrm{cm}): 30.261 \mathrm{~ms}$
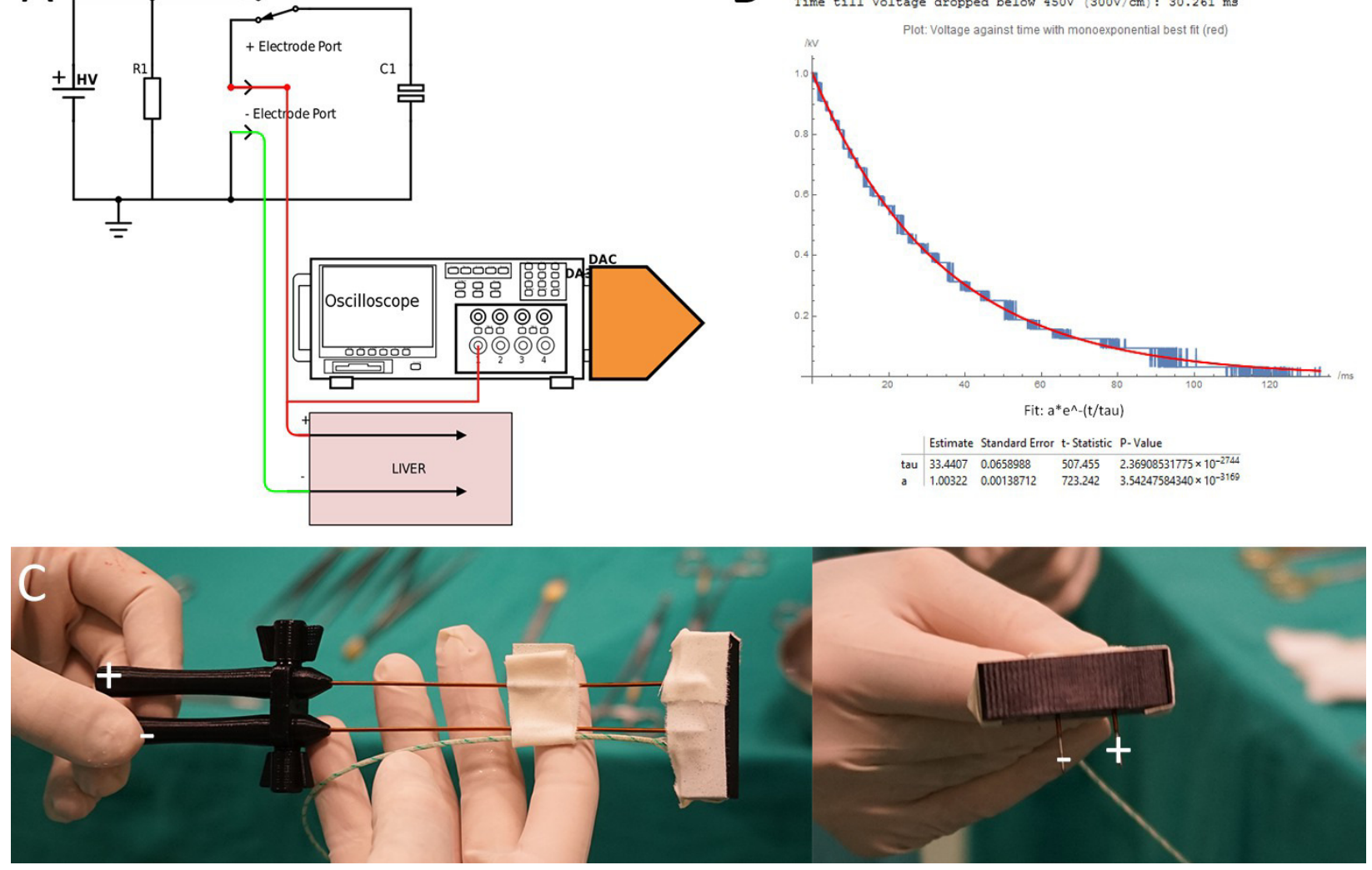

166 Figure 1: A. Generator and data acquisition schematic. B. An illustrative waveform applied in pig liver for $1671000 \mathrm{~V}$ and a time constant of $37 \mathrm{~ms}$ including statistical details of the measurement and fit from the 168 DAC. C. Needle configuration. 


\section{Mathematical Analysis}

170

171

172

173

174

175

176

177

178

179

180

181

182

183

184

185

186

187

188

189

190

191

192

193

194

195

196

197

198

199

200

201

202

203

204

205

206

207

The conductivity of the tissue in the model was adjusted to best fit the measured discharge curve of the capacitors of each pulse, which, since the charge of the capacity was defined, also defines the current. An illustrative waveform is shown in Fig. 1. While much more advanced mathematical models for electroporation and their thermal effects during pulse delivery are available (Corovic et al. 2013) we used a simplified model for several reasons. First, the available models do not have an exponential decay form yet. Second, electrolysis may substantially change the electrical and thermal parameters of the tissue, in a way that is not yet understood. Also there are not described effects on the metallic surface of the electrode changing conductivity. Therefore, we have used a simplified first order model which can provide the necessary information needed for this study. For future studies, the conductivity increase due to Joule heating and electroporation should be taken into account. The thermal and electrical field simulations in this study were performed using a finite element solver (Comsol Multiphysics 5.2) for the Laplace equation (electrical field) and Pennes Bioheat equation, in a way identical to that described in (Davalos et al. 2005) in a 3D model. The setup was approximated as two parallel titanium cylinders in a large volume of liver tissue with the parameters shown in Table 1. In case of discharging capacitors, the amount of Joules heating in tissue is prescribed by the dissipation of the charge energy, $\mathrm{Q},\left(\mathrm{Q}=\mathrm{C}^{*} \mathrm{U}_{0}\right)$. Therefore, specifying only, the initial voltage $\left(\mathrm{U}_{0}\right)$ and capacity (C) is sufficient to simulate the experiment. Thermal damage begins at temperatures higher than $42^{\circ} \mathrm{C}$, but only for prolonged exposures on the order of several seconds to hours. Damage is relatively low until $50-60^{\circ} \mathrm{C}$ at which the rate of damage dramatically increases (Diller 1992). Our interest was not in the distinction between these two processes, but only in the "worst case scenario" to estimate a radius of damage that could have been caused by thermal effects. We chose 30 seconds to look at a "worst case scenario" of tissue damage by heat to observe a lager distance from the electrode due to heat dissipation.

30 seconds appeared to be the worst case in terms of radius of possible thermal induced tissue necrosis when using the perfusion parameters as cited. The waveform delivered to the electrodes was assumed to be a perfect exponential decay in time, $t,\left(U=U_{0}{ }^{*} \exp -(t / \tau)\right)$, where $U_{0}$ is the initial voltage and the time constant is, $\tau$. The time constant was taken from the experimental data, through the analysis of the voltage trace during the delivery of the waveform.

\section{Results}

A series of 23 lesions were generated in experiments in which we studied the effects of the E2 waveform parameters on tissue ablation. The study examined the effects of the initial voltage, the time constant and the number of exponential decay voltage waveforms delivered. To facilitate a systematic and well defined analysis of the E2 phenomenon, we will focus on the results at midline between the two electrodes. The parameters chosen for this study were drawn from the experimental results of (Phillips, Krishnan et al. 2016) and are listed in Table 2. They were chosen to deliver substantial amounts of electrolytic products.

Lesion U E-field Distance Exposure C $\quad \tau \quad$ comment 


\begin{tabular}{|c|c|c|c|c|c|c|c|}
\hline \# & N & $/ \mathrm{V} / \mathrm{cm}$ & $/ \mathrm{mm}$ & $/ \mathrm{mm}$ & $/ \mu \mathrm{F}$ & $/ \mathrm{ms}$ & \\
\hline 1 & 750 & 500 & 15 & 10 & 100 & 50 & Figure $2 \mathrm{C}$ \\
\hline 2 & 750 & 500 & 15 & 10 & 100 & 100 & Figure 2D \\
\hline 3 & 1000 & 667 & 15 & 10 & 100 & 70 & Figure 3 \\
\hline 4 & $2 \times 1000$ & $2 \times 667$ & 15 & 10 & 100 & 79,92 & $\begin{array}{l}2 \text { pulses } 30 \text { s interval, same } \\
\text { polarity, Figure } 4+5\end{array}$ \\
\hline 6 & 1500 & 1000 & 15 & 20 & 100 & 70 & Figure $6+7$ \\
\hline
\end{tabular}

208 Table 2: Relevant parameters of the displayed experiments.
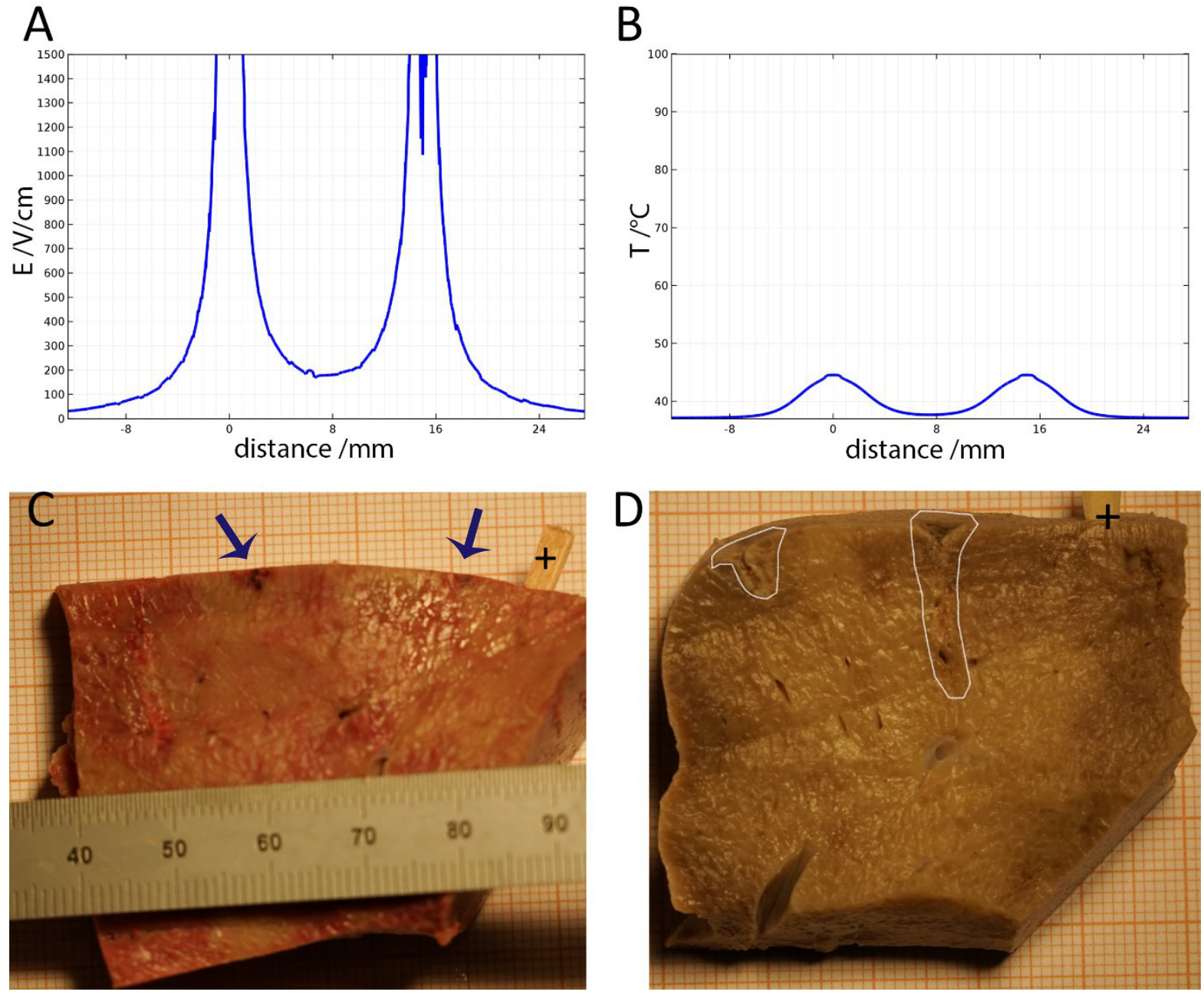

209 Figure 2: Study with an EDW with initial voltage difference between electrodes of $750 \mathrm{~V}$ and various time 210 constants. A. Calculated electric field. B. Calculated thermal field after 30 seconds. C. Macroscopic image 211 - 50 ms time constant - no ablation was noticed. D. Macroscopic image - 100 ms time constant - some 212 ablation near electrodes.

213 Figure 2 shows results from a series of studies in which the initial voltage between electrodes 214 was $750 \mathrm{~V}$, the distance between electrodes was $15 \mathrm{~mm}$, the exposed length was $10 \mathrm{~mm}$ and the 215 depth of penetration was $20 \mathrm{~mm}$. This configuration produces an initial voltage over distance of $216500 \mathrm{~V} / \mathrm{cm}$. The calculated electrical field norm is displayed in panel $\mathrm{A}$ and the calculated 
217 temperature in panel B. Geometrically, both graphs represent the $1 \mathrm{~d}$-cutline through the 218 perpendicularly induced electrodes with the electrical field and the temperatures respectively 219 on the $y$-axis. Panels $C$ and $D$ are the macroscopic histology from lesions treated with a voltage 220 of $750 \mathrm{~V}$ between the electrodes and time constants of $50 \mathrm{~ms}$ and $100 \mathrm{~ms}$, respectively. If tissue 221 resistance and conduction between electrode and tissue were constant, the discharge could be 222 fully described using the time constant of the EDW. However, secondary effects like thin layers of 223 burned tissue, can cause insulation and hence disrupt the ideal exponential decay. This does not 224 necessarily have any negative effect on the ablation, but will limit $\tau$ to adequately describe the 225 delivered waveform. The panels show the formalin embedded samples, sectioned in a plane that 226 is transverse to the centers of the two electrodes. In all the different experiments with $750 \mathrm{~V}$ 227 (500 V/cm voltage to distance between electrodes) there was no configuration in which the 228 lesion between electrodes became continuous. Panels $A$ and $B$ show that at the line midway 229 between the electrodes the electric field is less than $200 \mathrm{~V} / \mathrm{cm}$ and the temperature is below $23040^{\circ} \mathrm{C}$. 

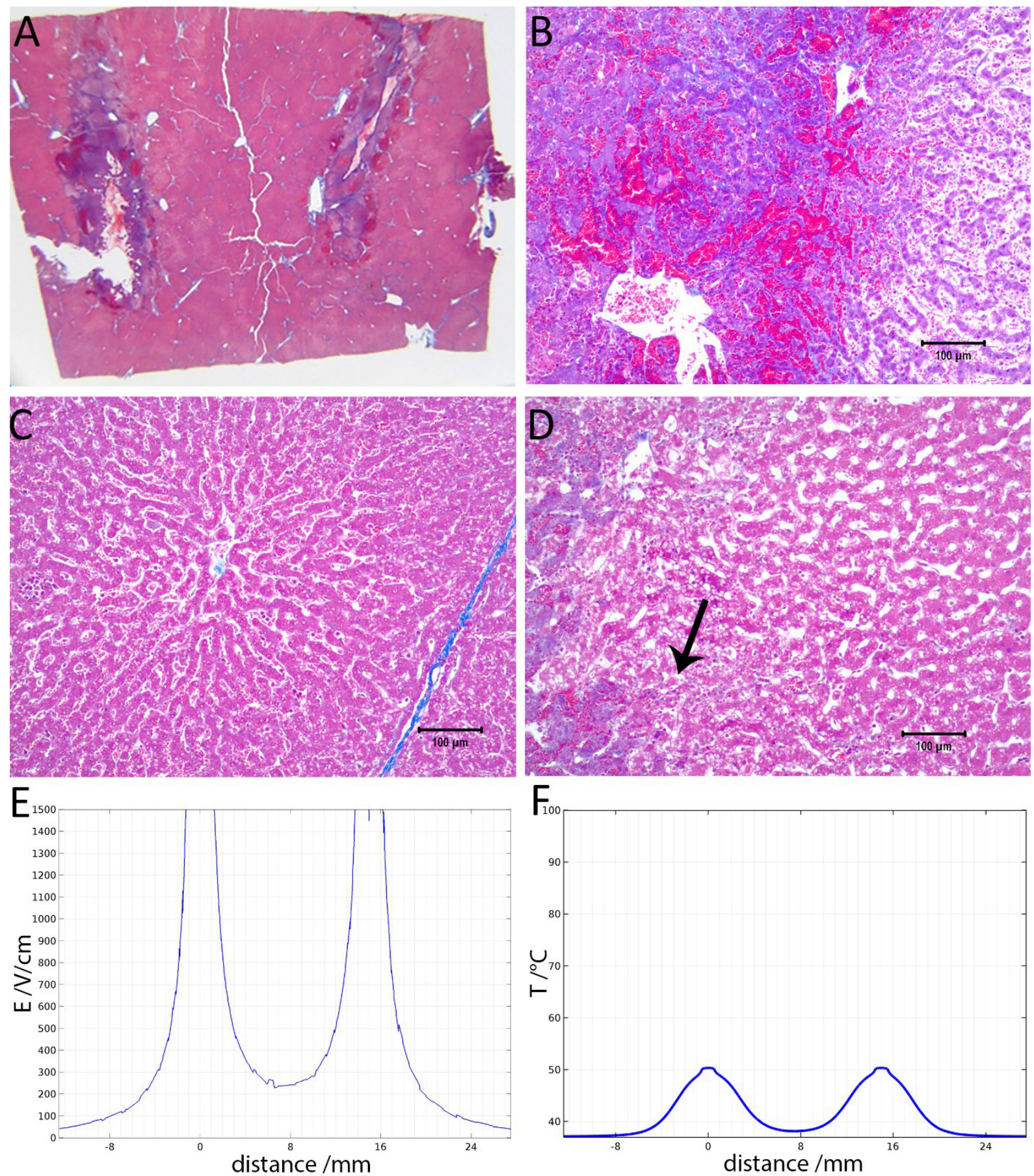

Figure 3: Study with one EDW with initial voltage difference between electrodes of $1000 \mathrm{~V}$ and time constant of $70 \mathrm{~ms}$. A. Histological slide with Masson's trichrome staining. B. 10x magnification of the right lesion, which is the anode. We see severe acute hepatocellular necrosis with coagulated blood (hemorrhage) in the sinusoids. C. 10x magnification between the electrodes. The cells do not appear to be affected. D. 10x magnification at the margin of the left lesion, which is the cathode. Here we see the borderline between the necrotic tissue on the left and partially affected cells on the right. E. Electric field strength distribution. F. Temperature distribution after 30 seconds (scale bar $100 \mu \mathrm{m}$ ). 
240 of penetration was $20 \mathrm{~mm}$ and the time constant was $70 \mathrm{~ms}$. The slides were prepared with 241 Masson's trichrome staining. Panel A gives an overview of the evaluated slide. The image is 242 taken in a plane that transverses the centers of the two electrodes. The area of the probe is 243 clearly visible, with a deep blue color at the site of the probes, representing the cellular damage 244 caused by thermal necrosis, surrounded by areas of coagulated blood (deep red color). 10x 245 magnification at the anode (Fig. 3B) illustrates an area of thermal necrosis, where the 246 hepatocytes have sustained more intense cellular ablation injury resulting in denaturation of the 247 cytoplasmic organelles. At the cathode (Fig. 3D) we can witness the gradual effect of the 248 treatment: Around the macroscopically visible lesion there is a pale area which represents less 249 affected cells immediately adjacent to the severely affected hepatocytes (marked with an 250 arrow). The sinusoidal spaces are dilated due to edema and/or hepatocellular swelling, while the 251 nuclei are condensed. The space between the electrodes is not fully ablated, as the microscopic 252 images show areas of unaffected cells (Figure $3 \mathrm{C}$ ). Figure 3E shows the calculated electric field 253 for a voltage of $1000 \mathrm{~V}$ and Figure 3F shows the calculated temperature distribution. Panels 3E 254 and $3 \mathrm{~F}$ show that, for these experimental conditions, the minimal electric field midway between 255 the electrodes is calculated to be about $240 \mathrm{~V} / \mathrm{cm}$ and the temperature midway between the 256 electrodes is well below $40^{\circ} \mathrm{C}$. 

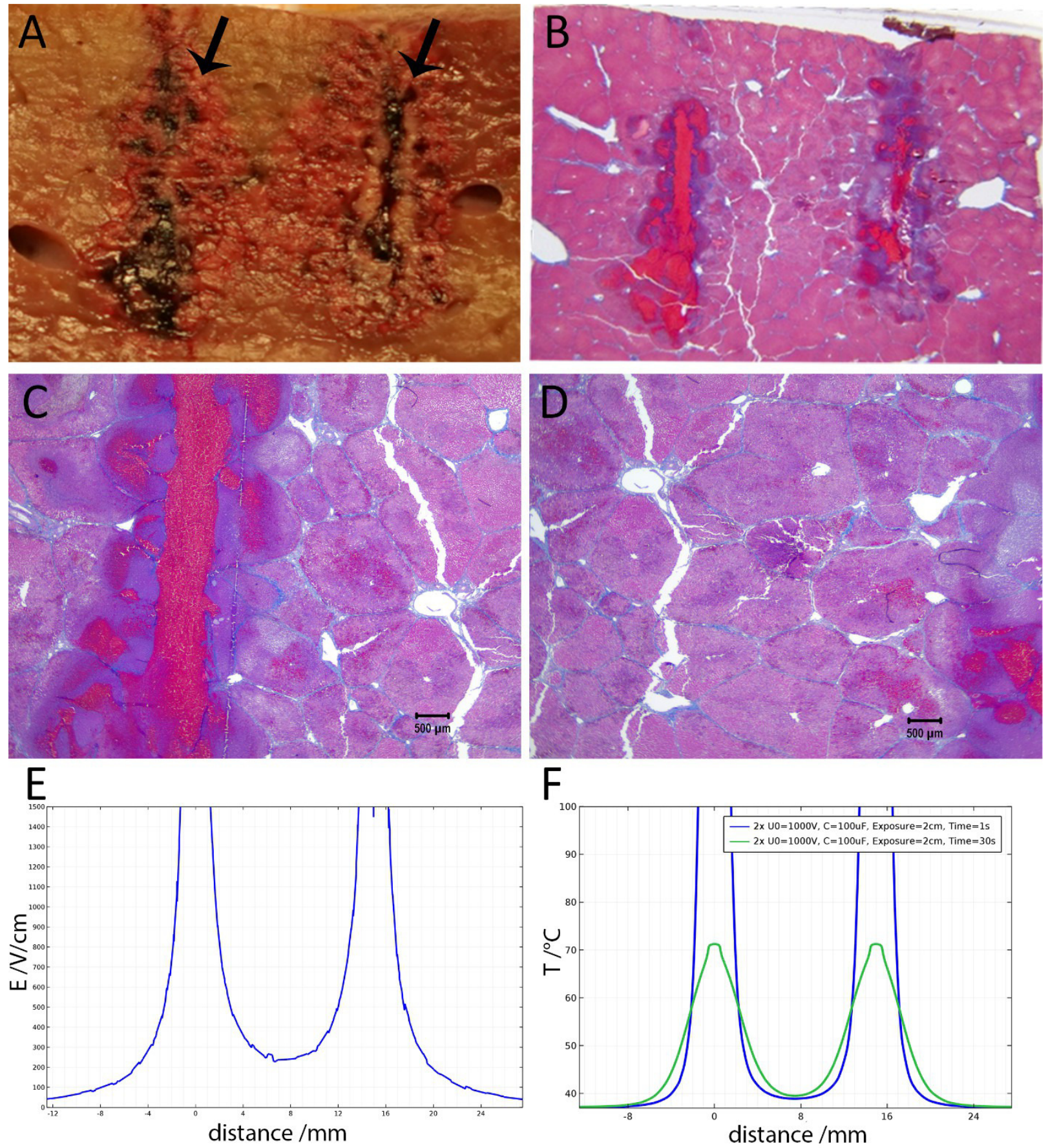

Figure 4: Study with 2 EDW separated by $30 \mathrm{~s}$ with time constants of 79 and $92 \mathrm{~ms}$, the first and second pulse respectively, $1000 \mathrm{~V}$ difference between electrodes placed at a distance of $15 \mathrm{~mm}$ between them, $10 \mathrm{~mm}$ exposed length, $100 \mu \mathrm{F}$ capacitor. Liver was extracted $18.5 \mathrm{~h}$ after treatment. A. Macroscopic histological slide (cathode left electrode anode right electrode) B. Masson's trichrome staining reveals blood coagulation (red) and ablation both around and in between electrodes. C. Close-up of the cathode, which is the left electrode. D. Close-up of the right electrode, which is the anode. (scale bar $500 \mu \mathrm{m}) \mathbf{E}$. Electric field strength distribution. F. Temperature distribution prior to the delivery of the second waveform at 1 and 30 seconds. 
266

267

268

269

270

271

272

273

274

275

276

277

278

279

280

281

282
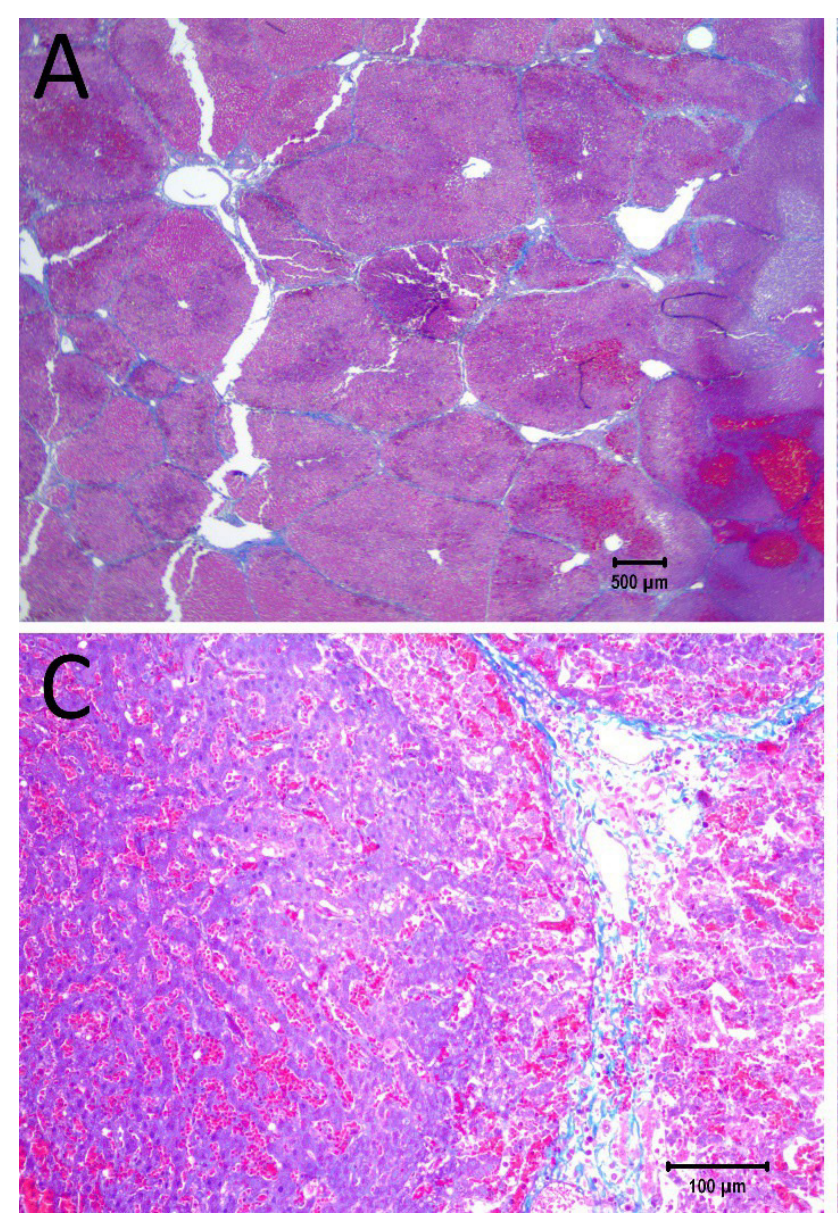

decay waveforms with similar parameters as those that produced Figure 3, were delivered at an interval of 30 seconds. The macroscopic image taken from a plane between the center of the two electrodes (Fig. 4A) shows that the partial electrode pathway (tunnel) is filled with coagulated blood. This is confirmed by the deep red linear region in the histological slides stained with Masson's trichrome staining in Figure 4B to 4D. The dark blue zone around that region (Figs 4B-4D) represents the more severely ablated hepatocytes, by virtue of being closest to the point of energy release. Figure $4 \mathrm{E}$ shows the calculated electric field for an exponential decay waveform with an initial voltage of $1000 \mathrm{~V}$ and Figure $4 \mathrm{~F}$ shows the calculated temperature distribution at the onset of the second pulse. There are two aspects to notice in panels $4 \mathrm{E}$ and $4 \mathrm{~F}$. Figure $4 \mathrm{E}$ is a copy of Figure 4E. It is obvious because we have used the electrical parameters of normal liver. However, it is known that the electrical conductivity of electroporated tissue changes after electroporation (Sel et al. 2005) (Ivorra and Rubinsky 2007), and therefore this panel may not be correct. We also anticipate that electrolysis will affect the electrical and thermal parameters in a way that is not fully known. The second aspect relates to the temperature distribution. Figure $4 \mathrm{E}$ shows that the calculated temperature distribution, when the second pulse is delivered is substantially elevated over the initial temperature when the first pulse is delivered, and thermal damage may be induced near the electrodes.
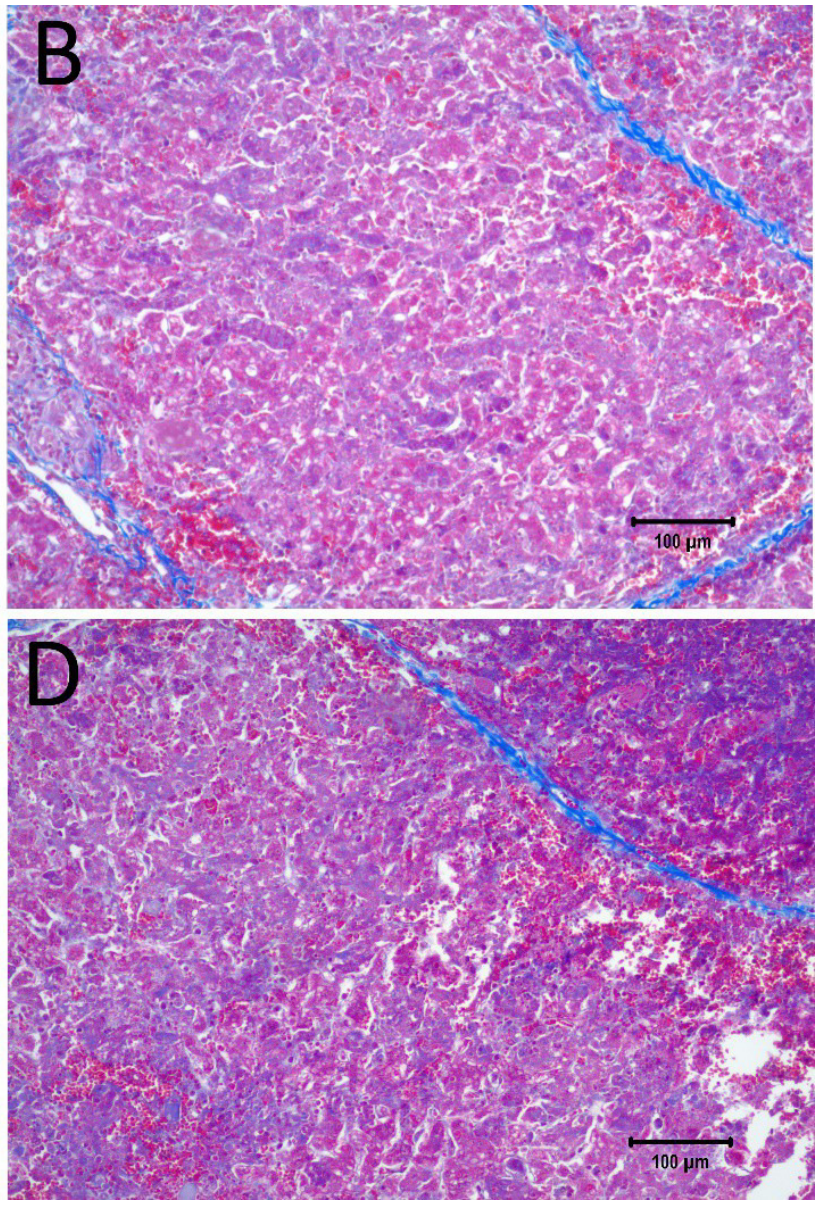

Figure 5: Details from Figure 4. A. Space between the electrodes in Figure 4. Bar indicates $500 \mu \mathrm{m}$. B. 10x magnification of cells between the electrodes, showing the details of the ablated area. C. 10x magnification of the cathode, showing edema and cellular ablation injury.

D. 10x magnification of the 
286 area by the anode, showing the margin of affected and non-affected cells. All images show Masson's 287 trichrome staining. Bars in B-D indicate $100 \mu \mathrm{m}$.

288 Figure 5 shows 10x magnified images of the histological slide from Figure 4. Fig. 5A shows the 289 space between the electrodes. Fig. 4B gives a 10x magnification of that area, showing a full 290 ablation zone, with affected cells throughout the area. Hepatocytes both at the cathode (Fig 5C) 291 and anode (Fig 5D) show condensed nuclei, with hemorrhage in the spaces between, however 292 with intact vessels (Fig 5C).

294 Figure 6 shows the histological results of exponential voltage profile in which the initial voltage 295 between electrodes was $1500 \mathrm{~V}$, the distance between electrodes was $15 \mathrm{~mm}$, the exposed 296 length was $20 \mathrm{~mm}$ and the depth of penetration was $30 \mathrm{~mm}$. It is important to notice that the 297 shown top $10 \mathrm{~mm}$ of the electrode was insulated. The slides were prepared with Masson's 298 trichrome staining. Fig. 6A shows the cells on the center line between the electrodes at the level 299 of the top $10 \mathrm{~mm}$ insulated part of the electrodes. Here we see that the cells are not affected by 300 the treatment. The next panel (Fig. 6B), however, shows the lesion which was caused by the 301 treatment in the uninsulated part of the tissue between the electrodes. The lesion is continuous 302 between electrodes at this level. Figure 6C displays the calculated electric field for a voltage of $3031500 \mathrm{~V}$, and Figure 6D shows the calculated temperature distribution. The electric field midway 304 between the electrodes is about $550 \mathrm{~V} / \mathrm{cm}$. The midway between electrodes temperature is 305 about $40^{\circ} \mathrm{C}$ and way above $50^{\circ} \mathrm{C}$ in proximity of the electrodes. 

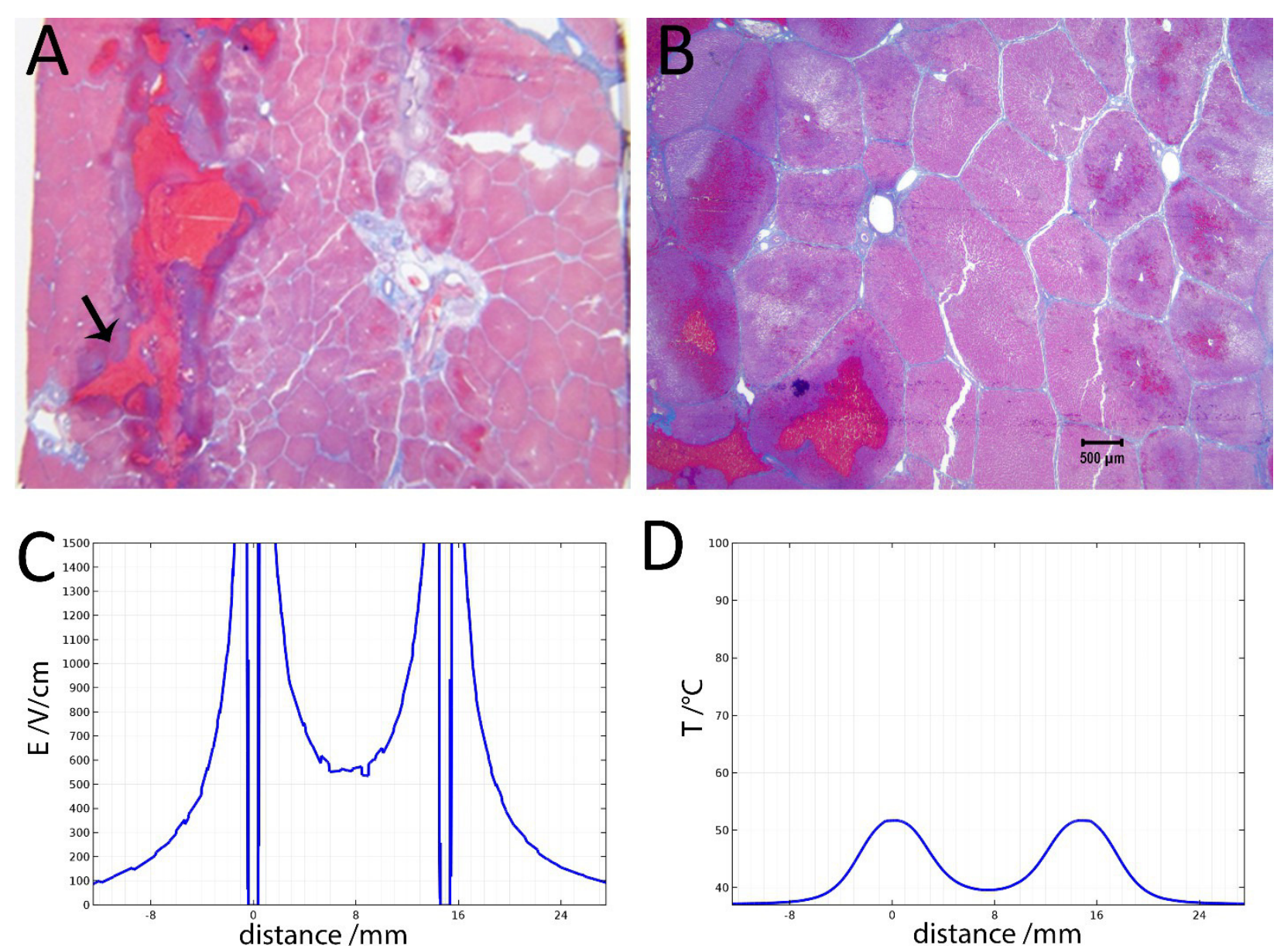

306

307

308

309

310

311

Figure 6: Study with a EDW with a time constant of $69 \mathrm{~ms}, 1500 \mathrm{~V}$ difference between electrodes placed at a distance of $15 \mathrm{~mm}$ between them, $200 \mathrm{~mm}$ exposed length, $100 \mu \mathrm{F}$ capacitor. A. Macroscopic cross section in a plane through the axis of the electrodes. Image taken between electrodes at the part where the electrodes were insulated, showing that the cells are not affected. B. Image taken between electrodes where the electrodes were not insulated showing that the lesion was bridged. (500 $\mu \mathrm{m} \mathrm{bar).}$ C. Electric field strength distribution. D. Temperature distribution after 30 seconds.

Figure 7 displays a 10x magnification of the pathological slide shown in Figure 6. Panel 7A is a magnification of the cathode, showing swollen and necrotic hepatocytes and a disrupted sinusoidal pattern. Between the electrodes (Fig. 7B) a bridged ablation with affected cells was observed, with a complete loss of cellular structure. At the anode (Fig. 7C) there is an affected cellular architecture with hemorrhage. Panels 7A-C show open and undamaged large blood vessels within the treatment field. 

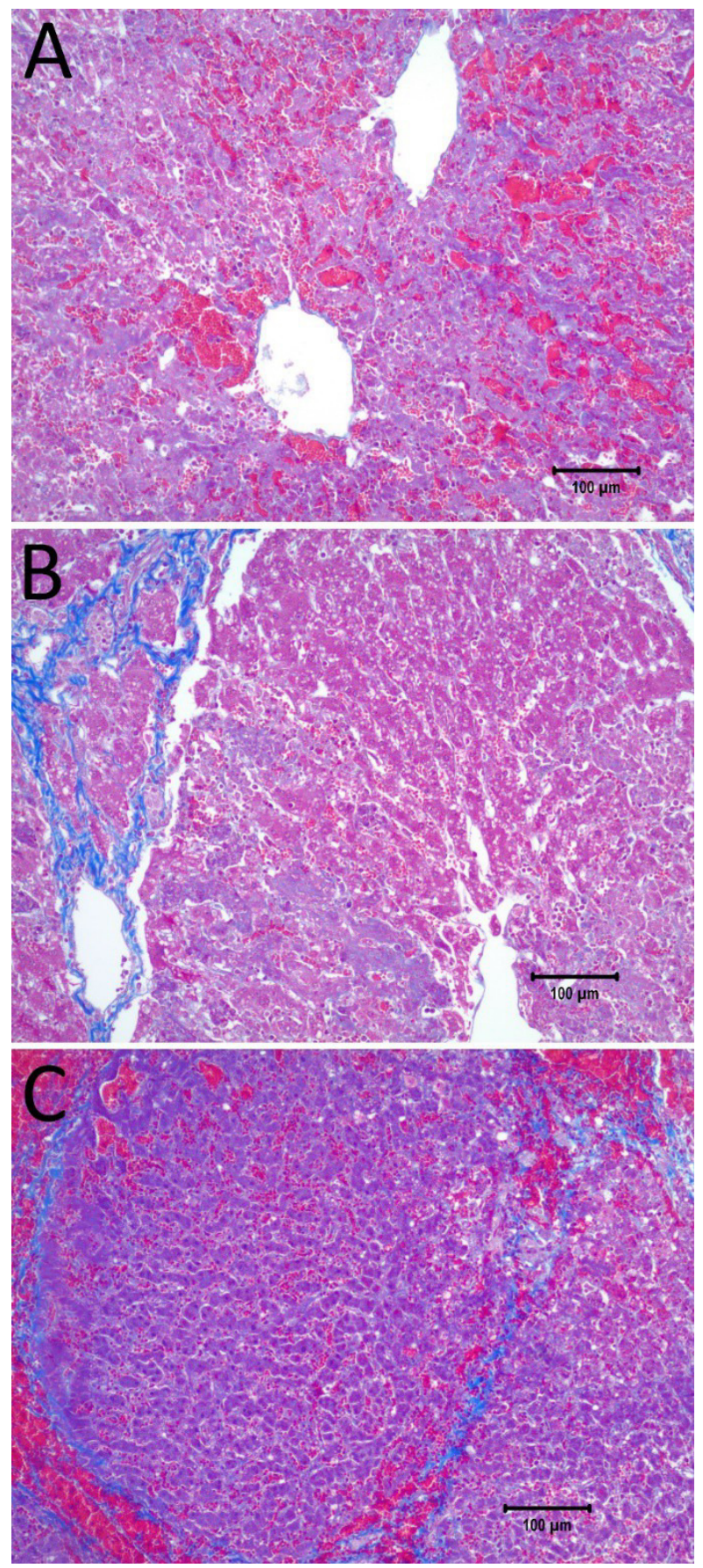

318 Figure 7: 10x magnification of the pathological slides shown in Fig 6. A. Image taken at the right 319 electrode, which was the cathode, showing necrotic, swollen hepatocytes and a disrupted sinusoidal 320 pattern. B. Image taken between the electrodes, illustrating a complete loss of cellular structure with 321 swollen hepatocytes. C. Left electrode, which was the anode, showing an affected cellular architecture 322 and hemorrhage. Note that the large blood vessels are open and unaffected. Scale bar $100 \mu \mathrm{m}$ 
324 Our main criteria for evaluating the exponential decay voltage waveform ability to ablate tissue 325 in a clinically significant manner was the ability to induce the ablation throughout the gap 326 between the electrodes. Therefore, the histological and mathematical analysis is focused on the 327 tissue found midway between the electrodes. This is the part of the treated tissue in which the lowest electric fields and lowest temperatures occur. Figures 2 and 3 show that there are parameters of initial voltage and time constant for which the tissue midway between the electrodes is not ablated. Figure 2A shows that for an initial voltage of $750 \mathrm{~V}$ and a distance of $1.5 \mathrm{~cm}$ between the electrodes $(500 \mathrm{~V} / \mathrm{cm}$ distance between electrodes), the electric field strength midway between the electrodes is lower than $200 \mathrm{~V} / \mathrm{cm}$. This value is substantially below the reversible electroporation threshold for the rabbit liver, which was measured to be $362+/ 21 \mathrm{~V} / \mathrm{cm}$ (Miklavcic et al. 2000). Since the calculated temperature midway between electrodes is below $40^{\circ} \mathrm{C}$, there is no mechanism to induce damage between the electrodes. The conditions in the region between the electrodes are below the levels required for irreversible electroporation ablation, reversible electroporation or thermal ablation.

Our experiment was designed to be performed without a muscle relaxant, however, in such a way as to allow for an immediate use of a muscle relaxant as soon as an undesirable level of muscle contraction is noted. From among the 23 experiments with the exponential decay voltage waveform done in this pig liver study, a muscle contraction requiring the use of a muscle relaxant (pancuronium) was detected in none. Two of our researchers (MS and PM) have experience with several hundred animal and human IRE procedures. On a scale from 1 to 5 , for IRE muscle contraction without muscle relaxants, they evaluate the contractions we observed as less than one. In comparison with muscle contractions when a muscle relaxant is used, they evaluated the contractions as similar. In fact, the muscle contraction was negligible, and at times unnoticeable. It should be emphasized that the reduction in muscle contraction is an expected benefit from the use of a single pulse. Reducing muscle contractions in IRE is an important area of current research. In particular, the HFIRE technology developed by Davalos and his group (Siddiqui, Latouche et al. 2016) and nanosecond pulses technology (Schoenbach, Hargrave et al. 2007). We believe that the effect we observed is related to the electrical discharge across electrolytically produced layer of gas around the electrodes. We have shown in (Guenther, Klein et al. 2015) that the electrical discharge across this layer of gas plays a major contribution to the observed violent motion of the electroporated object. We have also shown that this motion occurs primarily during the later pulses in a series of pulse experiments, when the electrolytically produced gas layer becomes substantial. Our single exponential decay pulse, while generating product of electrolysis, eliminates the violent electrical discharge across that gas layer. The violent discharge is eliminated, because by the time a large layer of gas has formed near the electrode, the potential at the electrode is below the value that can induce electrical breakdown. Obviously this observation is relevant only to the parameters used in this study, in which the maximal voltage was $1500 \mathrm{~V}(1000 \mathrm{~V} / \mathrm{cm}$ voltage over distance $)$ and the maximal time constant $148 \mathrm{~ms}$. 
365 electrodes is $1.5 \mathrm{~cm}$ and therefore the initial voltage to distance ratio is $750 \mathrm{~V} / \mathrm{cm}$. Figure $3 \mathrm{E}$ 366 shows that the electric field midway between electrodes is calculated to be below $300 \mathrm{~V} / \mathrm{cm}$. 367 This value is below the $362+/-21 \mathrm{~V} / \mathrm{cm}$ reversible electroporation threshold (Miklavcic et al. 368 2000). Tissue damage by heat can be also excluded, since the temperature between electrodes 369 does not exceed $40^{\circ} \mathrm{C}$ (Figure $2 \mathrm{~B}$ and $3 \mathrm{~F}$ ). In this case also, the conditions in the middle between 370 the electrodes are below the levels required for irreversible electroporation ablation, reversible 371 electroporation or thermal ablation.

372 Figures 4 and 5 show that it is possible to ablate the entire zone between electrodes by using 373 two consecutively delivered exponential decay waveforms with the same electrical parameters, 374 distance between the electrodes and waveform shape as those used to produce the results in 375 Figure 3. The initial voltage of the exponential decay waveform was $1000 \mathrm{~V}$, and as the distance 376 between the electrodes is $1.5 \mathrm{~cm}$, the initial voltage to distance ratio is $750 \mathrm{~V} / \mathrm{cm}$. Figure 3 shows that cells between the two electrodes survive the delivery of a single exponential decay waveform. The difference is that the results in Figure 3 were obtained with one exponential decay waveform and those in Figures 4 and 5 were obtained with two exponential decay waveforms. This suggests that the delivery of the second waveform is responsible for the cell death in the zone between the electrodes. Figures $3 \mathrm{E}$ and $4 \mathrm{E}$ show the theoretical calculated electric field. It is seen that in the zone between the electrodes the electric field is typical of reversible electroporation. This may explain why cells survive in the middle zone in Figure 3. There are a few possible explanations for the mechanism of cell death in the central zone between the electrodes after the delivery of two exponential decay waveforms. One explanation may be the change in electrical and biophysical properties as a consequence of the delivery of the first waveform and the attendant change in the electrical field that was actually delivered during the second waveform. Figure $4 \mathrm{~F}$ shows that the calculated temperature prior to the delivery of the second exponential waveform is elevated relative to that prior to the delivery of the first waveform. Elevated temperatures favor electroporation and may reduce its threshold (Polak et al. 2014). Furthermore, it is known that electroporation changes the electrical conductivity of tissue. While Figure 4E was obtained for the electrical conductivity of the normal liver, the second waveform may generate a somewhat modified electric fields, which may favor the cell death seen in the middle zone in Figures 4 and 5. An additional or another possible mechanism responsible for the difference in cell ablation between the cases depicted in Figures 3 and 4 may be related to the effect of electrolytic products. The second waveform, which is responsible for the cell ablation in the central zone in Figure 4, has delivered twice the level of electrolytic compounds than that delivered in the single waveform treatment whose results are depicted in Figure 3. It is possible that the enhanced cell death may be related to the increased amount of products of electrolysis, due to the second waveform and their synergistic effect with reversible electroporation. A combination of the effects of changes in properties and products of

402 electrolysis may be also responsible for the difference in extent of cell ablation between the results depicted in Figure 2 and 3. Obviously a more thorough study is needed to elucidate this 404 mechanism. An important aspect of this study would be a detailed mathematical model that 405 combines calculation of electric fields, temperature and effects of electrolysis. tissue ablation. Here, an increase of the exponential decay waveform initial voltage to $1500 \mathrm{~V}$ has produce ablated tissue between the electrodes. Calculations show that the electric field 
409 midway between the electrodes is about $550 \mathrm{~V} / \mathrm{cm}$ (Figure 6E). This value is below the 410 irreversible electroporation threshold for the rat liver $(637 \mathrm{~V} / \mathrm{cm}+/-43 \mathrm{~V} / \mathrm{cm}$ ) (Miklavcic et al. 411 2000), keeping in mind that this threshold depends on several parameters such as pulse shape, 412 number of pulses, pulse duration and configuration (Qin et al. 2012). The temperature midway 413 between electrodes is about $40^{\circ} \mathrm{C}$, (Figure 6F), which is below the threshold of thermal damage. 414 The mechanism of tissue ablation at the midpoint between electrodes is neither irreversible 415 electroporation nor thermal. The most likely possible mechanism is the synergistic effect of 416 electrolysis and reversible electroporation.

417 The E2 protocol requires a special waveform comprised of an exponential decay shape with a 418 steep decrease in voltage to values that will not induce an electrical discharge across the 419 electrolytically product near the electrodes and a longer low voltage tail, that can generate 420 sufficient products of electrolysis for the E2 ablation. To the best of our knowledge currently 421 available electroporation systems cannot deliver exponential decay waveforms with the desired, 422 electrolytic products generating time constants. To this end we have modified existing commercial designs (e.g. Gene Pulser Xcell ${ }^{\mathrm{TM}}$ Electroporation System, BioRad, Hercules, CA), as described in the methods and materials section. The key difference is the use of larger capacitance, in essentially the same circuit.

426

427

428

429

430

431

This is a first large animal study on the use of the synergy between electrolysis and reversible electroporation to enhance tissue ablation by electroporation. However, the E2 combination seems promising. It has the ability to create comparable clinically relevant areas of tissue ablation, in a much shorter period of time than irreversible electroporation, with lower voltages and single waveforms, without the need to inject drugs and without the need for paralyzing anesthesia.

\section{Acknowledgements}

433 We would like to thank Dr. Narayan Raju from Pathology Research Laboratory, Inc for his 434 assistance on the pathological examination and analysis.

\section{References}

439 Czymek, R., D. Dinter, S. Loeffler, M. Gebhard, T. Laubert, A. Lubienski, H.-P. Bruch and A. Schmidt 440 (2011). "Electrochemical Treatment: An Investigation of Dose-Response Relationships Using an 441 Isolated Liver Perfusion Model." Saudi Journal of Gastroenterology 17(5): 335-342.

442 Davalos, R. V., L. M. Mir and B. Rubinsky (2005). "Tissue ablation with irreversible 443 electroporation." Annals of Biomedical Engineering 33(2): 223-231.

444 Diller, K. R. (1992). Modeling of bioheat transfer processes at high

445 and low temperatures. Bioengineering Heat Transfer. Y. I. Choi. Boston, Academic Press: 157446 357. 
447 Emsley, J. (2001). "Titanium". Nature's Building Blocks: An A-Z Guide to the Elements. Oxford, 448 England, UK, Oxford University Press.

449 Guenther, E., N. Klein, P. Mikus, M. K. Stehling and B. Rubinsky (2015). "Electrical breakdown in 450 tissue electroporation." Biochemical and Biophysical Research Communications 467(4): 736-741.

451 Hamilton, W. A. and A. J. H. Sale (1967). "Effects of high electric fields on microorganisms. 2. 452 Mechanism of action of the lethal effect." Biochimica et Biophysica Acta 148: 789-800.

453 Ivorra, A. and B. Rubinsky (2007). "In vivo electrical impedance measurements during and after 454 electroporation of rat liver." Bioelectrochemistry 70(2): 287-295.

455 Maglietti, F., Michinski, S., Olaiz, N., Castro, M., Suárez, C., \& Marshall, G. (2013). The role of ph 456 fronts in tissue electroporation based treatments. PloS one, 8(11), e80167.

457 Marty, M., G. Sersa, J. R. Garbay, J. Gehl, C. G. Collins, M. Snoj, V. Billard, P. F. Geertsen, J. O. 458 Larkin, D. Miklavcic, I. Pavlovic, S. M. Paulin-Kosir, M. Cemazar, N. Morsli, Z. Rudolf, C. Robert, G. 459 C. O'Sullivan and L. M. Mir (2006). "Electrochemotherapy - An easy, highly effective and safe 460 treatment of cutaneous and subcutaneous metastases: Results of ESOPE (European Standard 461 Operating Procedures of Electrochemotherapy) study." EJC Supplements 4(11): 3-13.

462 Miklavčič, D., Šemrov, D., Mekid, H., \& Mir, L. M. (2000). A validated model of in vivo electric

463 field distribution in tissues for electrochemotherapy and for DNA electrotransfer for gene

464 therapy. Biochimica et Biophysica Acta (BBA)-General Subjects, 1523(1), 73-83.

465 Mir, L. M., M. Belehradek, C. Domenge, S. Orlowski, B. Poddevin, J. J. Belehradek, G. Schwaab, B. 466 Luboinski and C. Paoletti (1991). "Electrochemotherapy, a new antitumor treatment: first clinical 467 trial." Comptes Rendus de l'Academie des Sciences Serie III Sciences de la Vie 313: 613-618.

468 Mir LM, Banoun H, Paoletti C (1988) Introduction of definite amounts of nonpermeant 469 molecules into living cells after electropermeabilization: direct access to the cytosol. Exp Cell Res 470 175(1):15-25

471 Nilsson, E., H. von Euler, J. Berendson, A. Thorne, P. Wersall, I. Naslund, A. S. Lagerstedt, K. 472 Narfstrom and J. M. Olsson (2000). "Electrochemical treatment of tumours." 473 Bioelectrochemistry 51(1): 1-11.

474 Orlowski S, Belehradek J Jr, Paoletti C, Mir LM (1988) Transient electropermeabilization of cells in 475 culture. Increase of the cytotoxicity of anticancer drugs. Biochem Pharmacol 37(24):4727-4733

476 Phillips, M., H. Krishnan, N. Raju and B. Rubinsky (2016). "Tissue ablation by a synergistic 477 combination of electroporation and electrolysis delivered by a single pulse." Annals of 478 Biomedical Engineering.

479 Phillips, M., N. Raju, L. Rubinsky and B. Rubinsky (2015). "Modulating electrolytic tissue ablation 480 with reversible electroporation pulses." Technology 3(1): 45-53.

481 Polak, A., Tarek, M., Tomšic, M., Valant, J., Ulrih, N. P., Jamnik, A., Kramar, P., Miklavcic, D. (2014). 482 Electroporation of archaeal lipid membranes using MD simulations. Bioelectrochemistry, 100,

483 18-26.Qin, Z., Jiang, J., Long, G., Lindgren, B., \& Bischof, J. C. (2013). Irreversible electroporation: 484 an in vivo study with dorsal skin fold chamber. Annals of biomedical engineering, 41(3), 619-629. 485 Rubinsky, B., G. Onik and P. Mikus (2007). "Irreversible electroporation: A new ablation modality 486 - Clinical implications." Technology in Cancer Research \& Treatment 6(1): 37-48.

487 Sale, A. J. H. and W. A. Hamilton (1968). "Effects of high electric fields on microorganisms. 3. 488 Lysis of erythrocytes and protopasts." Biochimica et Biophysica Acta 163: 37-43.

489 Schoenbach, K. H., B. Hargrave, R. P. Joshi, J. F. Kolb, R. Nuccitelli, C. Osgood, A. Pakhomov, M. 490 Stacey, R. J. Swanson, J. A. White, S. Xiao, J. Zhang, S. J. Beebe, P. F. Blackmore and E. S. Buescher 491 (2007). "Bioelectric effects of intense nanosecond pulses." leee Transactions on Dielectrics and 492 Electrical Insulation 14(5): 1088-1109. 
493 Sel, D., Cukjati, D., Batiuskaite, D., Slivnik, T., Mir, L. M., \& Miklavcic, D. (2005). Sequential finite

494 element model of tissue electropermeabilization. IEEE Transactions on Biomedical Engineering, 495 52(5), 816-827.

496 Siddiqui, I. A., E. L. Latouche, M. R. DeWitt, J. H. Swet, R. C. Kirks, E. H. Baker, D. A. Iannitti, D. 497 Vrochides, R. V. Davalos and I. H. McKillop (2016). "Induction of rapid, reproducible hepatic 498 ablations using next-generation, high frequency irreversible electroporation (H-FIRE) in vivo." 499 Hpb 18(9): 726-734.

500 Stehling, M. K., E. Guenther, P. Mikus, N. Klein, L. Rubinsky and B. Rubinsky (2016). "Synergistic 501 Combination of Electrolysis and Electroporation for Tissue Ablation." Plos One 11(2).

502 Turjanski, P., Olaiz, N., Maglietti, F., Michinski, S., Suarez, C., Molina, F. V., \& Marshall, G. (2011). 503 The role of $\mathrm{pH}$ fronts in reversible electroporation. PloS one, 6(4), e17303. 